\title{
ANÁLISE DE COMPORTAMENTOS DE MÃES QUE ACOMPANHAM SEUS FILHOS DURANTE TRATAMENTO ODONTOLÓGICO
}

\author{
MOTHERS' BEHAVIOR ANALYSIS DURING DENTAL TREATMENT OF THEIR \\ CHILDREN \\ Antônio Bento A. de Moraes \\ UNIVERSIDADE ESTADUAL DE CAMPINAS, BRASIL \\ ÁDERSON L. COSTA JÚNIOR \\ UNIVERSIDADE DE BRASÍLIA, BRASIL \\ LAURA M. TOMITA \\ UNIVERSIDADE ESTADUAL DE CAMPINAS, BRASIL \\ GuSTAVO S. ROLIM \\ UNIVERSIDADE FEDERAL DE SÃO CARLOS, BRASIL
}

\begin{abstract}
RESUMO
O objetivo deste trabalho foi descrever e analisar os comportamentos de dez mães durante sessões seqüenciais de atendimento odontológico de seus filhos. Todas as sessões foram filmadas em videoteipe com marcas sonoras a cada 15 segundos, indicando os momentos em que foram efetuados os registros dos comportamentos. Um mapeamento geral do repertório de comportamentos permitiu apontar padrões comuns a todas as participantes e padrões específicos, indicadores de maior ansiedade de algumas participantes. Proporcionalmente, o procedimento de anestesia injetável evocou maior freqüência de comportamentos indicadores de fuga e/ou esquiva das mães. Os resultados permitem levantar a hipótese de que o cirurgião-dentista, atento aos comportamentos de pais e acompanhantes de crianças, pode adotar estratégias que reduzam a ansiedade dos pais, aumentando a freqüência de comportamentos colaborativos das crianças com o tratamento.

Palavras-chave: comportamentos de mães; ansiedade em odontologia; análise de comportamentos; odontopediatria.
\end{abstract}

\section{ABSTRACT}

The aim of this study was to describe and analyze the behavior of 10 mothers during sequential dental treatment sessions of their children. All sessions were recorded onto videotape with sonorous marks every 15second interval, indicating the moments that behavior records would be performed. A behavioral repertoire general plotting indicated some common and specific behavior patterns, which were indicators of anxiety for some participants. Proportionally, local anesthesia injection procedures evoked avoidance and mothers' escape behaviors. Results may establish the hypothesis that the pediatric dentistry, aware of parents' behaviors, can adopt strategies that reduce parents' anxiety, increasing children's collaborative behaviors.

Key words: Mother’s Behavior; Dental Anxiety; Behavior Analysis; Pediatric Dentistry.

O estudo do comportamento dos pais que acompanham seus filhos durante sessões de tratamento odontológico ainda constitui um tema controvertido. Nossa experiência clínica permite afirmar que a maior parte dos odontopediatras não costuma discutir com os pais como seus comportamentos podem ter maior ou menor influência sobre os comportamentos da criança durante a execução de procedimentos odontológicos. Por outro lado, observamos que uma pequena parcela de profissionais aborda o tema, em entrevista com os

1 Antônio Bento A. de Moraes é Professor Titular da Área de Psicologia Aplicada da Faculdade de Odontologia de Piracicaba da Universidade Estadual de Campinas, UNICAMP - abento@fop.unicamp.br; Áderson L. Costa Júnior - Professor Adjunto do Instituto de Psicologia da Universidade de Brasília - UnB - aderson@unb.br; Laura M. Tomita - Doutoranda em Odontologia, Área de Farmacologia, Anestesiologia e Terapêutica da Faculdade de Odontologia de Piracicaba da Universidade Estadual de Campinas - UNICAMP - lauratomita@yahoo.com.br; Gustavo S. Rolim - Psicólogo, Mestre em Educação Especial, Universidade Federal de São Carlos - UFSCAR - gurolim@caramail.com. 
pais, e ainda fornece instruções de conduta e suporte social durante as sessões. Contribuições da psicologia têm permitido análises mais detalhadas de comportamentos dos pais, no consultório de odontologia, e suas implicações para a continuidade do tratamento e da condição de saúde bucal das crianças (Cardoso, 2002; Moraes, Costa Junior \& Rolim, 2004).

Conforme descrevem Blount, Sturges e Powers (1990), crianças expostas a procedimentos médicos e odontológicos, especialmente de caráter invasivo, e seus acompanhantes, podem influenciar-se mutuamente durante a execução destes eventos, estabelecendo cadeias comportamentais que aumentam a probabilidade da evocação de respostas de enfrentamento eficiente e/ou de estresse. Para Blount, LandolfFritsche, Powers e Sturges (1991), reações comportamentais dos pais, em termos físicos, verbais e/ou fisiológicos, são facilmente percebidas pela criança, que tende a utilizá-las como condição estabelecedora para adoção de estratégias de colaboração, de concorrência ou de fuga às diversas situaçóes de tratamento de saúde. Uma expressão facial da mãe, por exemplo, percebida pela criança como típica de apreensão, aumenta a probabilidade de que a criança antecipe a ocorrência de um evento desagradável e não colabore com sua execução. $\mathrm{Da}$ mesma forma, comportamentos de baixa tolerância à situação e concorrentes com os eventos do tratamento podem configurar uma condição de ansiedade, dificultando, ou mesmo impedindo, a atuação de pediatras, odontopediatras e outros profissionais que lidam com a saúde de crianças (Costa Junior, 2001).

No que se refere aos pacientes, comportamentos indicadores de ansiedade, manifestados por crianças em atendimento odontológico, têm sido reconhecidos como o principal evento que dificulta a realização e a conclusão do tratamento (Aartman, van Everdingen, Hoogstraten, \& Schuurs, 1998). Estudos mais recentes ainda destacam que comportamentos típicos de ansiedade, não suficientemente compreendidos, ou desconsiderados por odontopediatras, são potenciais promotores de transtornos de comportamento mais amplos que podem, inclusive, generalizar-se para outras situações de tratamento de saúde (Baier, Milgrom, Russell, Mancl, \& Yoshida, 2004; Cardoso, Loureiro, \& Nelson-Filho, 2004). Uma das implicações mais importantes desta situação é a diminuição da probabilidade de que a criança venha a colaborar com outros tratamentos a que for exposta, caracterizando situações identificadas como típicas de medo (Moraes, Possobon, Costa Junior, \& Rolim, 2005).

Desde a década de 60 do século XX, pesquisas em odontopediatria e odontologia comportamental têm investigado a origem e o desenvolvimento da ansiedade no contexto do tratamento odontológico (Melamed \& Williamson, 1994). Embora ainda não tenha sido possível mapear, com exatidão, todas as variáveis relacionadas funcionalmente às manifestações de ansiedade de pacientes e acompanhantes, podemos afirmar que sua ocorrência aponta para uma complexa etiologia multifatorial. Muitos autores têm atribuído, há décadas, os episódios de ansiedade infantil à ansiedade demonstrada pela mãe que acompanha a criança ao consultório, apontando que esta relação pode resultar em maior probabilidade de comportamentos não-colaborativos da criança durante o atendimento em curso e em futuros tratamentos a que a criança será exposta (Allen \& Stokes, 1987; Johnson \& Baldwin, 1969; Klingberg \& Berggren, 1992; Milgrom, Mancl, 
King, \& Weinstein, 1995; Ramos-Jorge, Pordeus, Serra-Negra, \& Paiva, 1999; Wright, Alpern, \& Leake, 1973).

Entre estes trabalhos, alguns merecem comentários mais detalhados. O estudo clássico de Johnson e Baldwin (1968), por exemplo, investigou os comportamentos de crianças durante tratamento odontológico, relacionando-os com o nível de ansiedade materna. Os participantes foram 60 crianças, com idade entre três e sete anos, cujo tratamento odontológico envolvia, pelo menos, uma extração dental. Os resultados mostraram que filhos de mães com elevado nível de ansiedade demonstraram taxas significativamente maiores de comportamentos concorrentes com o tratamento quando comparadas às crianças com mães pouco ansiosas.

Wright e colaboradores (1973) investigaram os comportamentos de 124 crianças, de três a seis anos de idade, expostas pela primeira vez a tratamento odontológico, bem como os indicadores de ansiedade das mães. O objetivo era avaliar se uma explicação às mães sobre o procedimento a ser executado, antes do seu início, poderia reduzir a ansiedade materna, modificando, também, o repertório de comportamentos das crianças. Os resultados mostraram que as mães que receberam informações prévias evocaram, menos freqüentemente, comportamentos indicadores de ansiedade e foram mais eficientes na solicitação de colaboração das crianças durante o tratamento. Observou-se, também, uma redução significativa dos comportamentos concorrentes das crianças em relação ao tratamento.

Allen e Stokes (1987) utilizaram técnicas de reforçamento positivo, por meio da liberação de pequenos brindes e elogios, contingentes a comportamentos colaborativos de crian- ças de três a seis anos, expostas a tratamento odontológico. Além da redução nos níveis de não-colaboração com o tratamento, os autores identificaram uma diminuição na freqüência de comportamentos indicadores de ansiedade, manifestados pelos pais que acompanhavam seus filhos ao consultório. Os autores apontam que a redução na ocorrência de comportamentos não-colaborativos das crianças foi o resultado da combinação dos efeitos da estratégia de reforçamento e da diminuição dos níveis de ansiedade dos pais com o sofrimento da criança durante as sessōes.

Já na década de 90 do século XX, Klingberg e Berggren (1992) investigaram transtornos comportamentais em 99 crianças cujos pais apresentavam níveis elevados de medo de dentista e recusa freqüente de se submeterem a tratamento odontológico. Os resultados confirmaram a relação entre o medo dos pais e o medo apresentado por seus filhos na mesma situação, destacando, ainda, que as crianças de pais que apresentavam os níveis mais altos de medo tinham maiores índices de faltas e cancelamento de consultas e maior probabilidade de abandonar o tratamento sem concluí-lo.

Apesar do volume de estudos que apontam para a influência da manifestação de ansiedade da mãe sobre comportamentos nãocolaborativos da criança, pesquisas mais recentes, utilizando procedimentos metodológicos mais refinados, tecnologia de vídeo e análises funcionais de comportamento, têm identificado outras variáveis responsáveis pela manifestação de ansiedade da criança submetida a tratamento odontológico. Mais do que um comportamento indicador de ansiedade, evocado pela mãe, os estudos apontam para a relevância da experiência da criança com outros tratamentos odontológicos e de saúde em geral, percepção 
da criança acerca do tratamento, nível de autocontrole dos eventos do tratamento, nível educacional dos pais, importância atribuída pelos pais a práticas preventivas de saúde bucal e habilidades do cirurgião-dentista em manejar o comportamento da criança no consultório (Arnrup, Berggren, Brobeg, \& Bodin, 2004; Cardoso, Loureiro, \& Nelson-Filho, 2004; Klaassen, Veerkamp, \& Hoogstraten, 2003; Skaret, Kvale, \& Raadal, 2003).

No caminho oposto aos estudos que destacam a relação entre os comportamentos dos pais e dos filhos no consultório odontológico, alguns estudos não identificam relação entre a ansiedade manifestada pelos pais, no consultório, e os comportamentos de seus filhos. Folayan, Adekoya-Sofowora, Otuyemi e Ufomata (2002), por exemplo, estudaram 81 crianças, com idade entre oito e treze anos, sem experiência odontológica. Os resultados mostraram que o nível de ansiedade das mães foi significativamente maior que o nível de ansiedade dos pais. No entanto, níveis considerados elevados de ansiedade foram obtidos em apenas duas mães e um pai. Também não foi identificada correlação, estatisticamente significativa, entre os níveis de ansiedade das mães e dos pais com comportamentos indicadores de ansiedade entre as crianças. Resultados semelhantes foram obtidos por Ten Berge, Veerkamp, Hoogstraten e Prins $(2003 ;$ 2001), bem como por Klaassen, Veerkamp \& Hoogstraten (2003).

Um outro conjunto de estudos recentes aponta para a necessidade de que o cirurgiãodentista seja treinado e passe a utilizar, sistematicamente, instrumentos psicológicos que mapeiam comportamentos indicadores de ansiedade antecipatória e, desta forma, possa planejar o tratamento conforme o perfil comportamental de pacientes e acompanhantes (Baier \& cols., 2004; Goumans, Veerkamp, \& Aartman, 2004; Schwartz, 2005).

Um aspecto crítico, a ser considerado entre muitos dos trabalhos que se propóem a investigar a ansiedade materna no consultório de odontopediatria, é a limitação metodológica no que se refere: (a) ao reduzido número de sessões experimentais realizadas; (b) ao fato de que nem sempre as sessões são analisadas em sua totalidade; (c) à grande quantidade de dados obtidos a partir da percepção dos pais sobre sua própria ansiedade, referida em entrevistas pré e pós-sessão; e (d) aos poucos estudos que utilizam metodologia observacional direta para identificar comportamentos indicadores de ansiedade.

O objetivo deste trabalho foi descrever como se comportam as mães que acompanham seus filhos ao consultório, durante sessões consecutivas de tratamento odontológico, utilizando-se metodologia observacional direta de comportamentos.

\section{MÉTODO}

\section{Participantes}

Os participantes deste estudo foram 10 mães, que acompanharam seus filhos durante seis sessões seqüenciais de tratamento odontológico ao Centro de Pesquisa e Atendimento Odontológico para Pacientes Especiais (Cepae) da Faculdade de Odontologia de Piracicaba, da Universidade Estadual de Campinas (UNICAMP).

Todas as crianças tinham história de nãocolaboração com tratamento odontológico, conforme histórico de serviços de odontologia da cidade e/ou região há, no mínimo, seis meses. As crianças também tinham que apresentar um número mínimo de quatro molares cariados para 
que, da terceira à sexta sessão, cada dente recebesse a restauração necessária. Com o intuito de padronizar o tratamento, além do número de dentes afetados, também foi considerada a gravidade da lesão cariosa, aceitando-se apenas crianças com lesões de cárie que necessitassem de anestesia local para o tratamento.

\section{Procedimento}

As duas primeiras sessões tinham o objetivo de familiarizar a criança com o ambiente odontológico, equipamentos e profissionais presentes, sendo realizados apenas procedimentos de exame clínico e medidas profiláticas. Comportamentos colaborativos com o cirurgião-dentista eram, imediatamente, seguidos de reforçamentos positivos sociais.

Nas quatro sessões seguintes era realizado o tratamento odontológico restaurador (curativo). Em todas as sessões eram executados os mesmos procedimentos clínicos, seguindose uma rotina idêntica para todas as sessões: exame clínico, anestesia tópica, anestesia injetável, isolamento absoluto, preparo cavitário, restauração e encerramento.

Todas as sessões foram gravadas em fitas de videoteipe, com marcas sonoras a cada 15 segundos, indicativas dos momentos em que seriam efetuados os registros dos comportamentos das mães. As observações e registros foram realizados pelo mesmo pesquisador e por um observador independente, previamente treinado, que assistiam, separadamente, às sessôes gravadas e faziam o registro dos comportamentos das mães dentro de cada intervalo de 15 segundos. Também era registrado o procedimento clínico que estava sendo executado. Os registros foram, então, comparados e obteve-se índices de concordância superiores a $85 \%$.
Para a observação e registro dos comportamentos das mães, foram identificadas 16 categorias comportamentais, definidas operacionalmente e codificadas a partir de observações prévias das fitas gravadas. São elas: Acariciar/Tamborilar, Beijar, Consolar criança, Conter criança, Desviar olhar, Distrair criança, Instruir criança, Lamentar, Observar procedimento, Oferecer ajuda, Pegar no colo, Posicionar criança, Prometer recompensa, Questionar procedimento, Seguir instrução e Segurar mão. A definição operacional das categorias encontra-se no Anexo A.

$\mathrm{O}$ projeto de pesquisa foi submetido à apreciação do Comitê de Ética em Pesquisa da Faculdade de Odontologia de Piracicaba, da Universidade Estadual de Campinas, tendo sido aprovado pelo protocolo no $130 / 2002$. Os dados apresentados neste trabalho referem-se apenas às sessões de tratamento curativo.

\section{RESULTADOS E DISCUSSÃO}

Considerando-se as 40 sessões de tratamento odontológico analisadas neste estudo, observou-se que a categoria que mais ocorreu foi Acariciar/Tamborilar, conforme demonstrado pela Figura 1, registrada em todas as sessões. Embora as categorias Conter criança, Instruir criança, Observar procedimento, Posicionar criança e Segurar mão tenham sido observadas em todas as mães, pelo menos uma vez, não ocorreram em todas as sessões. As categorias Instruir criança e Observar procedimento foram observadas em 39 sessões e a categoria Conter criança foi registrada em 38 sessões.

Os comportamentos menos freqüentes foram Questionar procedimento, observado em duas mães (M2 e M10) e Lamentar, apresentado por apenas uma mãe (M2). Dentre as mães, 
M2 e M10 foram aquelas que demonstraram maior repertório comportamental em termos de freqüência de ocorrência de comportamentos, levantando-se, assim, a hipótese de que apresentavam maior grau de ansiedade, expresso em termos de respostas que indicavam sofrimento (Distress Behavior) e/ou necessidade de buscar mais informações por meio da observação dos procedimentos em execução e questionamentos ao cirurgiāo-dentista.

As Figura 2 apresenta a freqüência de respostas das categorias Consolar criança, Desviar olhar, Observar procedimento, Pegar no colo, Acariciar, Oferecer ajuda, Segurar mão e Conter criança de $\mathrm{M} 2$, permitindo comparar padrôes distintos de comportamento.

A hipótese de "ansiedade elevada" de M2 pode ser ressaltada, ainda, pelo registro da maior freqüência de ocorrência de Desviar olhar, durante todo o atendimento, especialmente em rotinas odontológicas apontadas como invasivas, tais como a anestesia injetável e o isolamento absoluto. Nestas rotinas, a duração do comportamento de Desviar olhar foi superior a $66 \%$ da duração destas rotinas, em todas as sessões, chegando a $85 \%$ de duração na última sessão.
No outro extremo, identificou-se M3, participante que apresentou as freqüências mais baixas de comportamentos, sendo a única a não apresentar as categorias Desviar olhar e Oferecer ajuda, registradas em todas as outras mães. Embora não tenha oferecido ajuda à criança, definida neste estudo como uma categoria de comportamento verbal, nem acompanhado visualmente os procedimentos odontológicos em execução, M3 apresentou, na terceira sessão, a maior freqüência de resposta para a categoria conter criança (superior a $73 \%$ da sessão) e a menor latência entre a ocorrência de movimentos físicos da criança e a emissão de contenção física, conforme ilustrado na Figura 3.

Nas demais sessões, esteve entre as três participantes com maior freqüência de ocorrência desta categoria de comportamento, com média superior a $28 \%$ da duração de cada sessão. Os dados obtidos permitem afirmar que $\mathrm{M} 3$ responde às exigências do tratamento, isto é, efetua a contenção da criança na primeira sessão curativa e apresenta modificaçóes gradativas no responder, passando, nas sessóes subseqüentes, a acariciar e segurar a mão da criança com maior freqüência.

As demais participantes (M1 e M4 a M9) apresentaram repertório de comportamentos

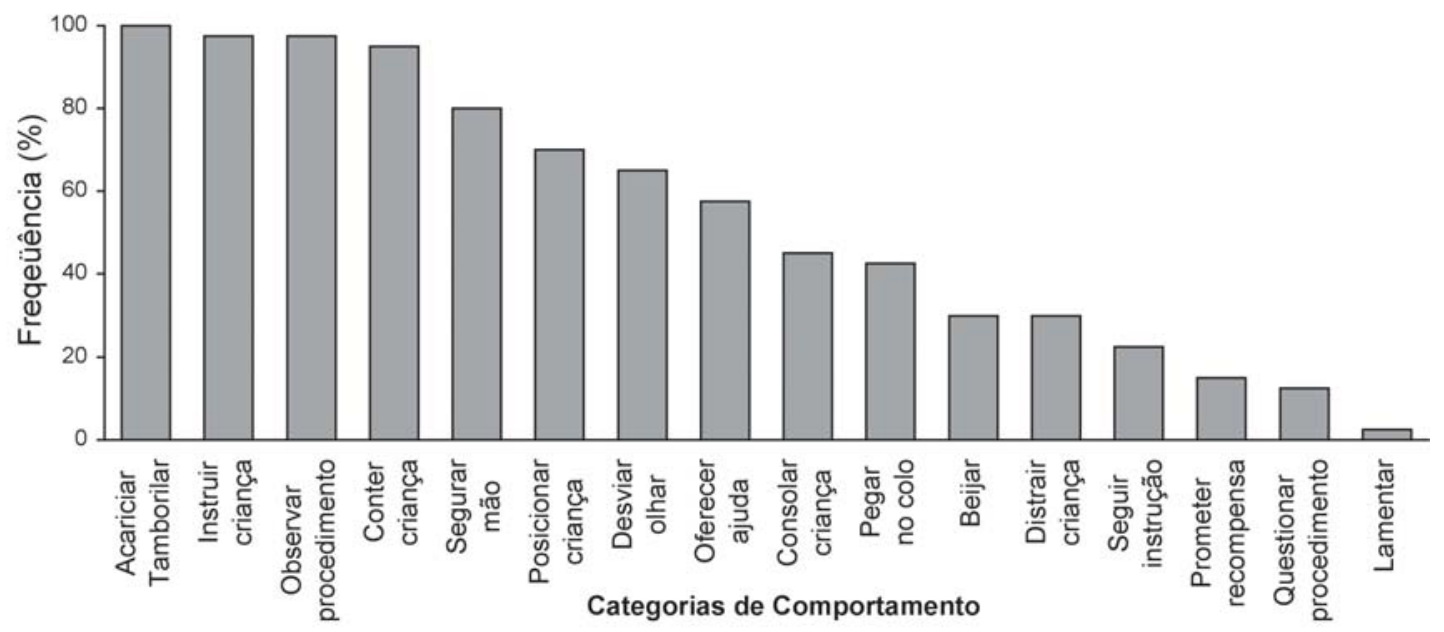

Figura 1. Freqüência de respostas de categorias de comportamento, considerando-se todas as participantes (M1 a M10). 
semelhantes, podendo-se sugerir que este seja um padrão comportamental adotado pela maioria das mães durante o atendimento odontológico de seus filhos. As categorias de comportamentos que ocorreram em alta freqüência (mais de 75\% das sessões), por exemplo, foram Instruir criança, Observar procedimento, Conter criança e Segurar mão, comportamentos comumente esperados de acompanhantes de crianças submetidas a tratamento odontológico.

A Figura 4 apresenta a freqüência de respostas de todas as participantes durante as quatro sessōes curativas, e permite verificar a evolução do repertório de comportamentos ao longo do tratamento. Observou-se que para as categorias Conter criança, Oferecer ajuda e Pegar no colo, a maior freqüência ocorreu na terceira sessão, isto é, a primeira sessão de tratamento curativo. Para estas categorias, verificou-se uma diminuição gradual de freqüência da terceira para a sexta sessão. A maior redução de freqüência foi registrada para a categoria Conter criança, que de $52,2 \%$ de ocorrência na terceira sessão, caiu para $11,5 \%$ na sexta sessão. Já a categoria Acariciar/Tamborilar obteve maiores médias de freqüência na quarta e quinta sessões. As demais categorias de comportamento mantiveram uma freqüência estável ao longo das quatro sessões.

A Figura 5 mostra o aumento e diminuição graduais, ao longo das sessões, da freqüência de resposta das categorias Segurar a mão e Conter a Criança, respectivamente. A primeira variou de $3,6 \%$ a $34 \%$ e a segunda de $50 \%$ a $15 \%$ da terceira à sexta sessão.

A Figura 6 apresenta a freqüência de resposta da categoria Desviar olhar, ao longo de cada procedimento odontológico clínico, durante as quatro sessões de tratamento curativo. Pôde-se notar que as maiores freqüên-

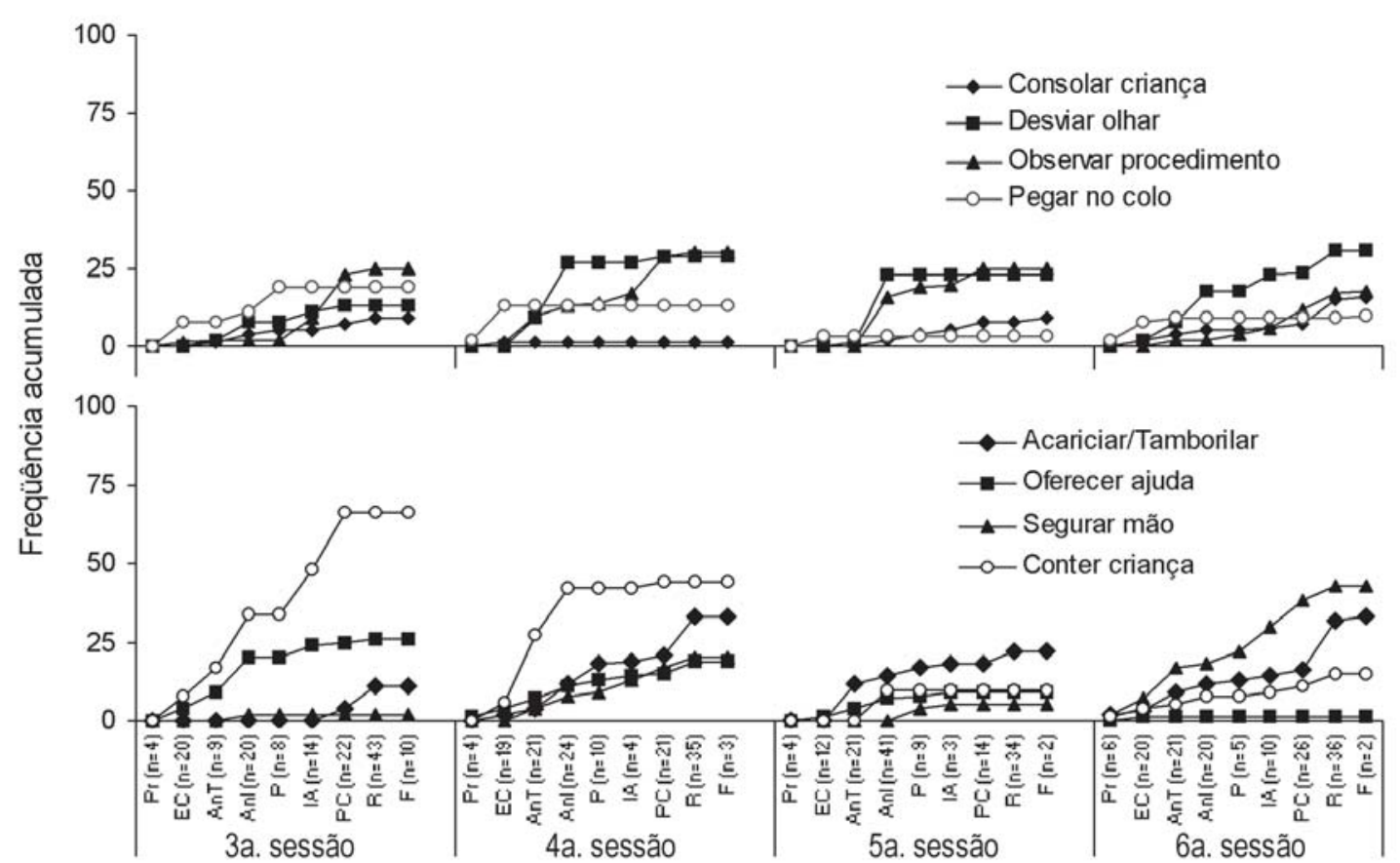

Figura 2. Freqüência de respostas de M2 para as categorias de comportamento Consolar criança, Desviar olhar, Observar procedimento, Pegar no colo Painel superior, Acariciar, Oferecer ajuda, Segurar mão e Conter criança Painel inferior, da terceira à sexta sessōes (Pr: Preparo; EC: Exame Clínico; AnT: Anestesia Tópica; AnI: Anestesia Infiltrativa; P: Pausa; IA: Isolamento Absoluto; PC: Preparo Cavitário; R: Restauração; F: Final). 
cias de ocorrência dessa categoria ocorreram durante os procedimentos de Anestesia Injetável e Isolamento Absoluto.

\section{DISCUSSÃO}

Os comportamentos das mães relacionados a Posicionar criança, Instruir criança e Conter criança são necessários para que o atendimento odontológico prossiga e fizeram parte do repertório comportamental de todas as mães estudadas. Em contingências de tratamento odontológico regular, especialmente durante a execução de rotinas invasivas, as mães são solicitadas a colaborar, apresentando estas respostas e sendo reforçadas socialmente. Estes comportamentos são facilitadores da execução do tratamento odontológico e, eventualmente, pode-se supor que sejam negativamente reforçados. Como também, as respostas de Instruir criança, Posicionar criança e Conter criança levam ao inicio compulsório da sessão e estabelecem uma diminuição do tempo de duração da sessão. Se um maior número de odontopediatras adquirisse o hábito de discutir com os pais, antes de iniciar o tratamento, como seus comportamentos podem influenciar o comportamento da criança durante as sessões, provavelmente, observaríamos uma maior freqüência de comportamentos colaborativos dos pais sem que o odontopediatra tivesse que solicitá-los enquanto executa rotinas invasivas.

M2 e M10 foram consideradas as mães com maior freqüência de comportamentos indicadores de ansiedade. $\mathrm{O}$ fato de comportamentos indicadores de ansiedade terem sido identificados, particularmente, em apenas duas participantes pode estar relacionado com as duas sessões iniciais de atendimento, nas quais não ocorriam procedimentos curativos. Tais sessões foram utilizadas para familiarizar as crianças e

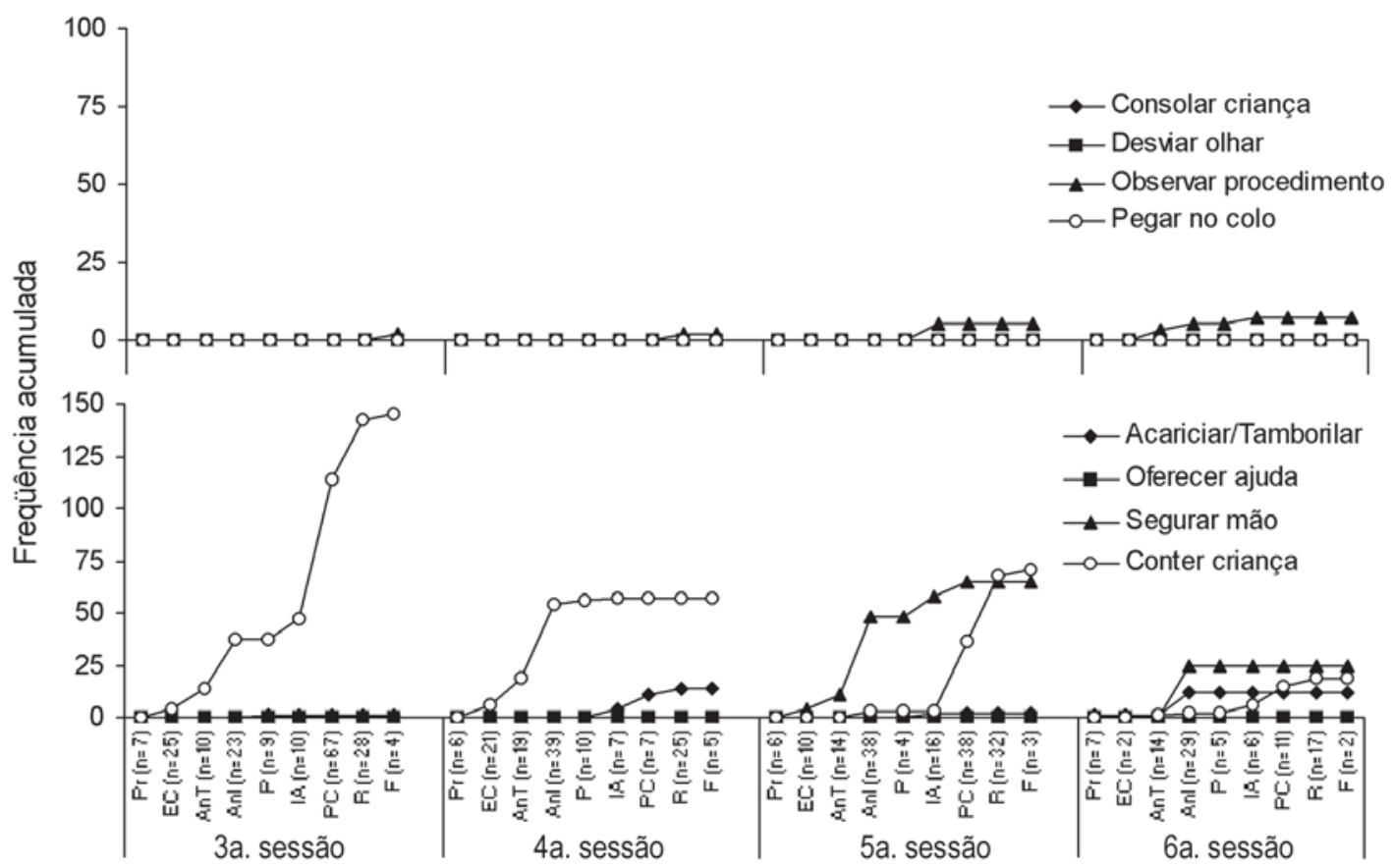

Figura 3: Freqüência de respostas de M3 para as categorias de comportamento Consolar criança, Desviar olhar, Observar procedimento, Pegar no colo Painel superior, Acariciar, Oferecer ajuda, Segurar mão e Conter criança Painel inferior, da terceira à sexta sessões (Pr: Preparo; EC: Exame Clínico; AnT: Anestesia Tópica; AnI: Anestesia Infiltrativa; P: Pausa; IA: Isolamento Absoluto; PC: Preparo Cavitário; R: Restauração; F: Final). 


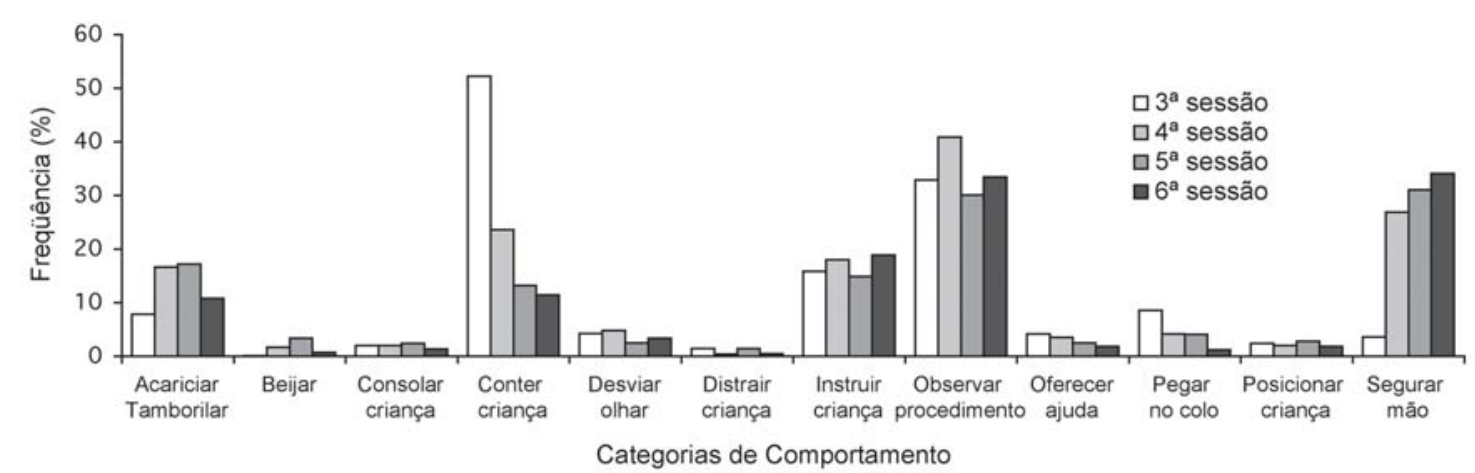

Figura 4: Freqüência de ocorrência das categorias de comportamento (M1 a M10) ao longo das quatro sessões de tratamento odontológico.

as mães com o ambiente físico do consultório, os equipamentos e o cirurgião-dentista, sendo realizados, apenas, procedimentos profiláticos e não invasivos. Sessões preparatórias têm sido utilizadas em diversos estudos com objetivo de reduzir a aversividade percebida em relação a ambientes de tratamento médico e odontológico (Bragado \& Fernandéz, 1997; Costa Junior, 2001; Possobon, 2003). Moraes e colaboradores (2005), no entanto, discutem eventuais efeitos adversos de sessóes preparatórias, que quando realizadas em número maior que o necessário, podem atuar como reforçadoras aos comportamentos de nãocolaboração da criança.

O baixo repertório comportamental de M3 poderia indicar, aparentemente, uma mãe pouco responsiva aos eventos do ambiente, incluindo os procedimentos odontológicos que estavam sendo executados na criança. A análise dos comportamentos de $\mathrm{M} 3$, no entanto, não confirmaram esta hipótese. Destaca-se que o baixo repertório comportamental de M3 não implicou em baixa frequiência de respostas. Esta participante mostrou-se extremamente atenta à necessidade de conter fisicamente a criança durante os episódios de não-colaboração com o tratamento. A responsividade da mãe, atenta à contenção física, garantiu que a criança apresentasse poucos movimentos físicos que exigissem a interrupção da atuação do profissional, possivelmente, agilizando a sessão e diminuindo o tempo de exposição de seu filho aos procedimentos odontológicos específicos de cada rotina.

A alta freqüência da categoria Tamborilarl Acariciar pode indicar que as mães, além da própria presença física, percebida como um fator de segurança para a criança, conforme Brazelton e Greenspan (2002), bem como Klaus, Kennel e Klaus (2000), também apresentam, sistematicamente, um estímulo afetivo reforçador, representado pelo toque e pelo carinho à criança, em todas as sessōes de tratamento, o que pode constituir um fator facilitador ao enfrentamento de eventos percebidos como estressantes (Costa Junior, 2001).

A maior freqüência de ocorrência, na primeira sessão de tratamento curativo, das categorias Conter criança, Oferecer ajuda e Pegar no colo, e a diminuição gradual das mesmas da terceira para a sexta sessão foi acompanhado por decréscimo da freqüência de comportamentos de não-colaboração das crianças, observado ao longo das sessôes de atendimento. Especialmente em relação à categoria Conter criança, Allen e Stokes (1987), Stark e colaboradores (1989) e Ten Berge e colaboradores (1999), observam que sua redução, ao longo de sessões de atendimento 


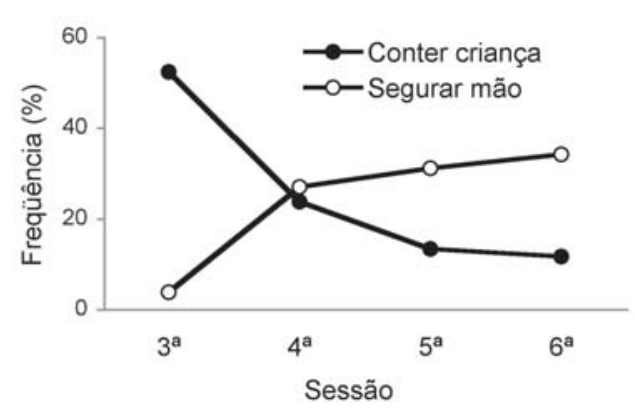

Figura 5: Freqüência de ocorrência das categorias de comportamento Conter criança e Segurar mão ao longo das quatro sessôes de tratamento de todas as participantes (M1 a M10).

Também para Cardoso (2002), a aplicação da Anestesia Injetável, a execução de extração/ cirurgia dentária e o uso do motor de altarotação (durante a rotina de Preparo Cavitário) foram os procedimentos mais freqüentemente considerados como difíceis para as crianças, sendo percebidos como ameaçadores ou incômodos pelos pais.

O comportamento das mães, de Desviar olhar, especialmente durante a execução da Anestesia Injetável, pode ser analisado como uma resposta de esquiva, alternativa adotada pelas participantes para "evitar" o próprio sofrimento. Além disso, estudos que investigam os aspectos aversivos da situação odontológica encontraram índices elevados de nãocolaboração das crianças durante os procedimentos de Anestesia Injetável (Yanase, Braham, Fukuta, \& Kurosu, 1996; Ram, Mamber, Chosack, \& Fuks, 1999).

A ocorrência de comportamentos como Desviar olhar, Questionar procedimento e Lamentar, por exemplo, poderiam constituir estímulos discriminativos para que o cirurgiãodentista disponibilizasse informação e esclarecimentos, permitindo que pais e acompanhantes expressassem suas preocupações e se tornassem participantes mais ativos do processo de tratamento odontológico de seus filhos. Ramos, Carrara e Gomide (2005), por exemplo, destacam a necessidade de que o cirurgiãodentista informe previamente aos pais e/ou acompanhantes sobre procedimentos odontológicos e técnicas de manejo comportamental que poderão ser utilizados durante o atendimento à criança. Tal cuidado pode reduzir, significativamente, a apreensão dos pais acerca de eventuais sofrimentos a que seus filhos serão expostos. A partir dos dados obtidos neste estudo, considerando-se que o comportamento de Desviar olhar pode indicar

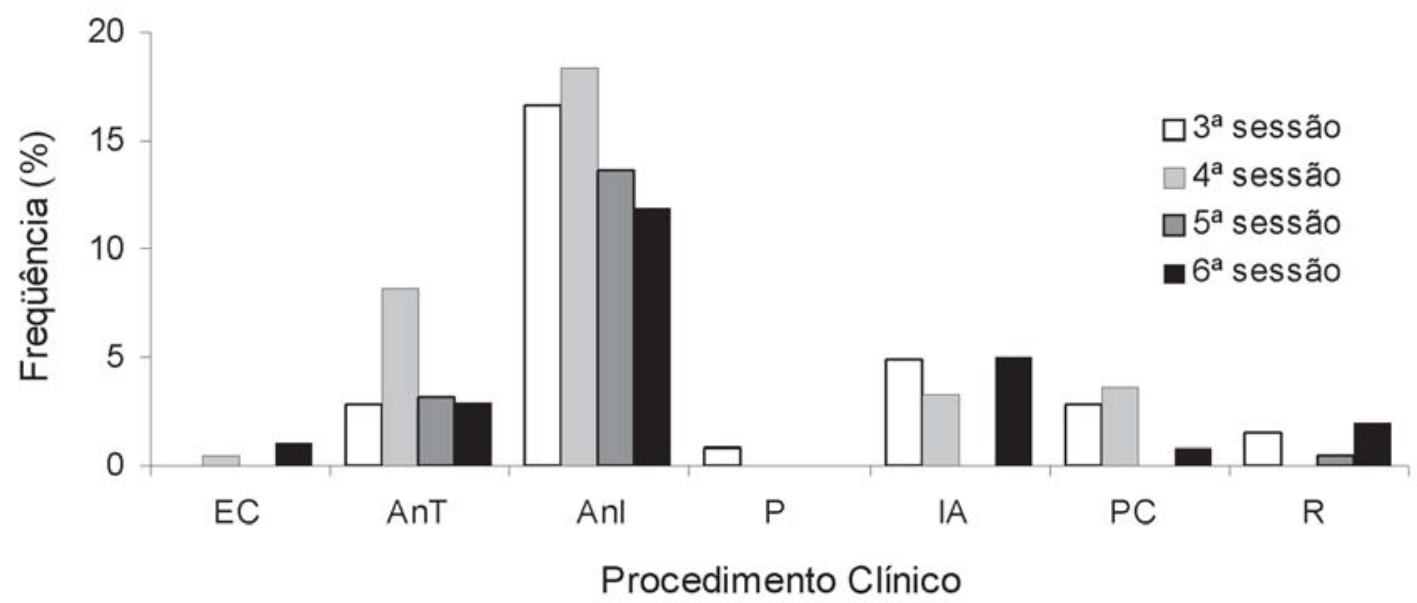

Figura 6: Freqüência de ocorrência da categoria de comportamento Desviar olhar, ao longo de cada procedimento clínico durante as quatro sessốes, considerandose todas as participantes - M1 a M10 (EC: Exame clínico; AnT: Anestesia Tópica; AnI: Anestesia Injetável; P: Pausa; IA: Isolamento Absoluto; PC: Preparo Cavitário; R: Restauração). 
odontológico, está diretamente relacionada ao aumento dos níveis de colaboração da criança, reduzindo-se a necessidade da execução de contenção física pela mãe.

A categoria Acariciar/Tamborilar obteve maiores médias de freqüência na quarta e quinta sessões, e foi também seguido pela redução da freqüência de ocorrência da categoria Conter criança. Para Blount e colaboradores (1990), observa-se que uma redução na ocorrência de comportamentos não-colaborativos, por parte de crianças submetidas a sessões consecutivas com procedimentos invasivos, geralmente é seguida pelo aumento de comportamentos indicadores de carinho e atenção dos acompanhantes que presenciam a execução dos procedimentos invasivos.

A diminuição da freqüência da categoria Conter criança e o aumento da freqüência da categoria Segurar mão (conforme ilustrado pela Figura 5) sugere que a categoria Segurar mão poderia ser considerada uma modalidade de contenção física preventiva (Possobon, 2003). Assim, diminuindo-se a necessidade $\mathrm{da}$ execução de contenção física da criança para a realização do tratamento, o comportamento de Segurar mão pode ter diminuído a probabilidade de movimentos bruscos da criança, além da função de incentivá-la a enfrentar o tratamento (Costa Junior, 2001).

A maior freqüência da categoria Desviar olhar durante a execução da rotina de Anestesia Injetável sugere que esta resposta esteja relacionada à percepção de aversividade do procedimento e/ou à crença da mãe quanto ao sofrimento que tal procedimento possa proporcionar à criança. Tal resultado é coerente com o obtido pelo estudo de Possobon (2003), no qual a Anestesia Injetável foi a rotina que gerou maior nível de ansiedade nas crianças. uma condição de apreensão da mãe e aumentar a probabilidade de uma resposta de nãocolaboração da criança com o tratamento, a adoção de estratégias educativas pelo cirurgiãodentista, tal como proposto por Ramos e colaboradores (2005), poderia criar uma condição de tratamento na qual a mãe não tivesse motivo para desviar o olhar da criança durante a execução de procedimentos clínicos considerados aversivos.

A realização deste estudo permitiu a descrição do repertório comportamental de mães que acompanham seus filhos com história de nãocolaboração a atendimento odontológico, em sessões de tratamento bucal. Pôde-se notar que algumas mães demonstram maior nível de ansiedade do que outras, mas existem certos comportamentos que são comuns a todas elas. Segundo Borges (1999), cada mãe possui um padrão pessoal de responder a situações estressantes, adquirido ao longo de sua história de vida, e mantido por inúmeros fatores ambientais, o que permite sugerir que os odontopediatras atentos aos comportamentos de pais e acompanhantes podem auxiliá-los a atuarem como agentes estimuladores de comportamentos colaborativos das crianças em tratamento.

\section{REFERÊNCIAS}

Aartman, I.H.A., van Everdingen, T., Hoogstraten, J., \& Schuurs, A.H.B. (1998). Self-report measurements of dental anxiety and fear in children: A critical assessment. Journal of Dentistry for Children, 65, 252258.

Allen, K.D., \& Stokes, T.F. (1987). Use of escape and reward in the management of young children during dental treatment. Journal of Applied Behavior Analysis, 20, 381-390.

Arnrup, K., Berggren, J., Brobeg, A.G., \& Bodin, L. 
(2004). A short-term follow-up of treatment outcome in groups in uncooperative child dental patients. European Journal of Paediatric Dentistry, 5, 216-224.

Baier, K., Milgrom, P., Russel, S., Mancl, L., \& Yoshida, T. (2004). Children's fear and behavior in private dentistry practices. Paediatric Dentistry, 26, 316-321.

Blount, R.L., Sturges, J.W., \& Powers, S.W. (1990). Analysis of child and adult behavioral variations by phase of medical procedure. Behavior Therapy, 21, 33-48.

Blount, R.L., Landolf-Fritsche, B., Powers, S.W., \& Sturges, J.W. (1991). Differences between high and low coping children and between parent and staff behaviors during painful medical procedures. Journal of Pediatric Psychology, 16, 795-809.

Borges, L.M. (1999). Treinamento de respostas de enfrentamento e colaboração em mães de crianças submetidas a procedimentos médicos invasivos. Dissertação de Mestrado, Universidade de Brasília. Brasília-DF.

Bragado, C.A., \& Fernandéz, A.M. (1997). Psychological treatment of evoked pain and anxiety by invasive medical procedures in pediatric oncology. Spain Journal of Psychology, 1, 17-36.

Brazelton, T.B., \& Greenspan, S.I. (2002). As necessidades essenciais da infância. Porto Alegre: Artes Médicas.

Cardoso, C.L., Loureiro, S.R., \& Nelson-Filho, P. (2004). Pediatric dental treatment: Manifestations of stress in patients, mother and dental school students. Pesquisa Odontológica Brasileira, 18, 150-155.

Cardoso, C.L. (2002). Tratamento odontopediátrico no contexto de uma clínica-escola: avaliação do estresse da criança, do acompanhante e do aluno. Tese de Doutorado, Universidade de São Paulo, Ribeirão Preto-SP.

Costa Junior, A.L. (2001). Análise de comportamentos de crianças expostas à punção venosa para quimioterapia. Tese de Doutorado, Universidade de Brasília, Brasília-DF.
Folayan, M.O., Adekoya-Sofowora, C.A., Otuyemi, O.D., \& Ufomata, D. (2002). Parental anxiety as a possible predisposing factor to child dental anxiety in patients seen in a suburban dental hospital in Nigeria. International Journal of Paediatric Dentistry, 12, 255-259.

Goumans, C., Veerkamp, J.S., \& Aartman, I.H. (2004). Dental anxiety and behavioral problems: What is their influence on the treatment plan? European Journal of Paediatric Dentistry, 5, 15-18.

Johnson, R., \& Baldwin, D.C. (1969). Maternal anxiety and child behavior. Journal of Dentistry for Children, 36, 87-92.

Johnson, R., \& Baldwin, D.C. (1968). Relationship of maternal anxiety to the behavior of young children undergoing dental extraction. Journal of Dentistry for Children, 47, 801-805.

Klaassen, M., Veerkamp, J., \& Hoogstraten, J. (2003). Predicting dental anxiety and the clinical value of anxiety questionnaires: An explorativa study. European Journal of Paediatric Dentistry, 4, 171176.

Klaus, M.H., Kennel, J.H., \& Klaus, P.H. (2000). Vinculo: construindo as bases para um apego seguro e para a independência. Porto Algre: Artes Médicas. Trabalho publicado originalmente em 1995.

Klinberg, G., \& Berggren, U. (1992). Dental problem behaviors in children of parents with severe dental fear. Sweden Dentistry Journal, 16(1/2), 27-32.

Melamed, B.G., \& Williamson, D.J. (1994). Programs for the treatment of dental disorders. Em: J.J. Sweet, R.H. Rozensky, \& S.M. Tovian (Orgs.), Handbook of Clinical Psychology in Medical Settings (pp. 539565). N. York: Plenum Press.

Milgrom, P., Mancl, L., King, B., \& Weinstein, P. (1995). Origins of childhood dental fear. Behavior Research and Therapy, 33, 313-319.

Moraes, A.B.A., Costa Junior, A.L. \& Rolim, G.S. (2004). Medo de dentista: ainda existe? Em M.Z.S. Brandão (Org.), Sobre comportamento e Cognição- 
Estendendo a psicologia comportamental e cognitiva aos contextos da saúde, das organizaçôes, das relaçôes pais e filhos e das escolas (pp. 171-178). Santo André, SP: Esetec.

Moraes, A.B.A., Possobon, R.F., Costa Junior, A.L., \& Rolim, G.S. (2005). Contingências aversivas em serviços de saúde. Em H.J. Guilhardi \& N.C. de Aguirre (Orgs.), Sobre Comportamento e Cognição Expondo a variabilidade (pp. 83-94). Santo André, SP: Esetec.

OMS - Organização Mundial da Saúde (1993). Classificação de Transtornos Mentais e de Comportamento da CID-10. Porto Alegre: Artes Médicas.

Possobon, R.F. (2003). Efeitos do diazepam sobre os comportamentos não-colaborativos de crianças em atendimento odontológico. Tese de Doutorado, Universidade Estadual de Campinas, Piracicaba-SP.

Ram, D., Mamber, E., Chosack, A., \& Fuks, A.B. (1999). The effect of metoclopramide and hydroxyzine in sedation of infants undergoing dental treatment. Journal of Dentistry for Children, 66, 49-52.

Ramos, M.M., Carrara, C.F., \& Gomide, M.R. (2005). Parental acceptance of behavior management techniques for children with clefts. Journal of Dentistry for Children, 72, 74-77.

Ramos-Jorge, M.L., Pordeus, I.A., Serra-Negra J.M.C., \& Paiva, S.M. (1999). A ansiedade materna como fator de influência na adaptação comportamental do paciente odontopediátrico. Arquivos de Odontologia, 35(1/2), 61-70.

Schwartz, B. (2005). Errors in dentistry: A call for apology. Journal of The American College of Dentistry,
72(2), 26-32.

Skaret, E., Kvale, G., \& Raadal, M. (2003). General self-efficacy, dental anxiety and multiple fears among 20-years-olds in Norway. Scandinavian Journal of Psychology, 44, 331-337.

Stark, L.J., Allen, K.D., Hurst, M., Nash, D.A., Rigney, B., \& Stokes, T.F. (1989). Distraction: Its utilization and efficacy with children undergoing dental treatment. Journal of Applied Behavior Analysis, 22, 297-307.

Ten Berge, M. Veerkamp, J.S., Hoogstraten, J., \& Prins, P.J. (2003). Childhood dental fear in relation to parental child-pearing attitudes. Psychological Reports, 92, 43-50.

Ten Berge, M. Veerkamp, J.S., Hoogstraten, J., \& Prins, P.J. (2001). Parental beliefs on the origins of child dental fear in the Netherlands. Journal of Dentistry for Children, 68, 51-54.

Ten Berge, M., Veerkamp, J.S., \& Hoogstraten, J. (1999). Dentists' behavior in response to child dental fear. Journal of Dentistry for Children, 66, 36-40.

Wright, G.Z., Alpern, G.D., \& Leake, J.L. (1973). The modifiability of maternal anxiety as it relates to children's cooperative dental behavior. Journal of Dentistry for Children, 40, 265-271.

Yanase, H., Braham, R.L., Fukuta, O., \& Kurosu, K. (1996). A study of the sedative effect of homeadministered oral diazepam for the dental treatment of children. International Journal of Paediatric Dentistry, 6, 13-17.

Submetido em 19 de abril de 2006 Aceito em 20 de dezembro de 2006 


\section{Anexo A}

Definição Operacional das Categorias de Comportamento das Mães

\begin{tabular}{|c|c|}
\hline Categoria & Definição \\
\hline Acariciar/Tamborilar & $\begin{array}{l}\text { Passar levemente a palma, costas ou os } \\
\text { dedos da mão sobre parte do rosto, cabelo ou } \\
\text { corpo da criança ou tocar com a mão, rápida e } \\
\text { sucessivamente, parte do corpo da criança, } \\
\text { mantendo ritmo constante ou ainda estando } \\
\text { com a mão apoiada em parte do corpo da } \\
\text { criança, movimentar suavemente a mão sem } \\
\text { retirá-la do local. }\end{array}$ \\
\hline Beijar & $\begin{array}{l}\text { Tocar lábios unidos e protusos em } \\
\text { alguma parte do corpo ou rosto da criança, } \\
\text { com ou sem estalido. }\end{array}$ \\
\hline Consolar criança & $\begin{array}{l}\text { Tentar aliviar ou suavizar a aflição, o } \\
\text { sofrimento da criança. Por exemplo: "não fique } \\
\text { assim", “ô meu amor". }\end{array}$ \\
\hline Conter criança & $\begin{array}{l}\text { Manter a(s) mão(s) parada(s), sobre ou } \\
\text { em volta de parte(s) do corpo da criança, } \\
\text { exercendo pressão e impedindo ou } \\
\text { interrompendo seus movimentos, como flexão } \\
\text { de pernas e braços e toque no profissional ou } \\
\text { em objetos utilizados no procedimento. }\end{array}$ \\
\hline Desviar olhar & $\begin{array}{l}\text { Imediatamente antes ou durante a inserção } \\
\text { da agulha no corpo da criança, fechar os olhos, } \\
\text { podendo fazer movimentos de pressão da } \\
\text { pálpebra superior sobre a inferior; virar a cabeça } \\
\text { para o lado oposto ao procedimento realizado } \\
\text { ou abaixar a cabeça, olhando em outra direção. }\end{array}$ \\
\hline Distrair criança & $\begin{array}{l}\text { Dirigir à criança uma frase afirmativa ou } \\
\text { interrogativa ou conversar sobre assuntos não } \\
\text { relacionados ao procedimento ou, cantar e } \\
\text { tentar desviar a atenção da criança do } \\
\text { procedimento que está sendo realizado. }\end{array}$ \\
\hline
\end{tabular}


Anexo A (CONTINUAÇÃo)

\begin{tabular}{|c|c|}
\hline Instruir criança & $\begin{array}{l}\text { Dizer para a criança o que ela deve fazer. } \\
\text { Por exemplo: "abra a boca", "coloque as mãos } \\
\text { para baixo", "não se mexa". }\end{array}$ \\
\hline Lamentar & $\begin{array}{l}\text { Manifestar, por meio de palavras, o seu } \\
\text { sofrimento. Por exemplo: "ai, meu Deus do } \\
\text { céu". Queixa, acompanhada de gemidos, } \\
\text { lamúria. }\end{array}$ \\
\hline Observar procedimento & $\begin{array}{l}\text { Olhar atentamente em direção ao } \\
\text { procedimento que está sendo realizado na criança. }\end{array}$ \\
\hline Oferecer ajuda & $\begin{array}{l}\text { Manifestar verbalmente apoio à criança. } \\
\text { Por exemplo: "a mamãe está aqui com você" ou } \\
\text { "posso fazer alguma coisa por você??. }\end{array}$ \\
\hline Pegar no colo & $\begin{array}{l}\text { Suspender a criança até a altura do } \\
\text { tronco, segurando seu corpo ou parte dele, e } \\
\text { aproxima-la de si, de modo que esta fique } \\
\text { sustentada pelos braços e apoiada em parte do } \\
\text { corpo da mãe. }\end{array}$ \\
\hline Posicionar criança & $\begin{array}{l}\text { Segurando o corpo da criança em qualquer } \\
\text { posição inicial, coloca-la sobre uma superfície, } \\
\text { de modo que todo o seu corpo fique em contato } \\
\text { com esta, ou mudar a criança de posição e } \\
\text { acomodá-la sobre a cadeira odontológica, de } \\
\text { modo a facilitar a atuação do profissional. }\end{array}$ \\
\hline Prometer recompensa & $\begin{array}{l}\text { Assegurar à criança a ocorrência de um } \\
\text { evento agradável, após a realização do } \\
\text { procedimento. }\end{array}$ \\
\hline Questionar procedimento & $\begin{array}{l}\text { Questionar o profissional em relação a } \\
\text { algum aspecto do procedimento. }\end{array}$ \\
\hline Seguir instrução & $\begin{array}{l}\text { Atender solicitação do profissional, como } \\
\text { conter a criança ou conversar com a criança. }\end{array}$ \\
\hline Segurar mão & $\begin{array}{l}\text { Encostar a(s) palma(s) da(s) mão(s) na mão } \\
\text { da criança, flexionando os dedos em torno dela. }\end{array}$ \\
\hline
\end{tabular}

